\title{
Le Théâtre du Vécu
}

\section{Jean Martin}

Dr méd., membre de la rédaction

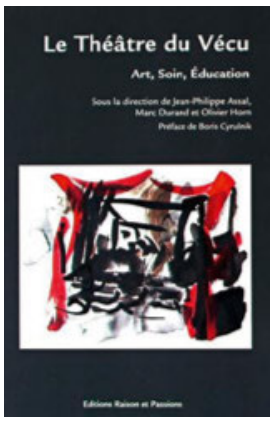

Le professeur genevois Jean-Philippe Assal, endocrinologue, est un pionnier reconnu dans le domaine de l'éducation du malade [1]. Il a développé avec son équipe des démarches très originales, qu'il enseignait notamment lors des stages extra-muros en montagne. Son dernier ouvrage présente une modalité mise en œuvre depuis une quinzaine d'années, le Théâtre du Vécu. Ceci avec une quinzaine de contributeurs: un tiers de médecins, un tiers issus des sciences de l'éducation et de la psychologie; un tiers du domaine du théâtre et de la communication. Cinq parties traitant de: genèse et structure de la démarche; vécus et témoignages; expériences faites dans d'autres lieux et cultures; intrication/complémentarité du soin, de l'art et de l'éducation (dimension anthropologique); recommandations pratiques.

Le Théâtre du Vécu, né d'une rencontre entre Assal et le metteur en scène Marcos Maldavia, a été conçu pour aider patients et soignants à mieux percevoir le poids affectif de la maladie chronique; la méthode a été introduite dans plusieurs pays d'Europe et quelques-uns outremer, dans les soins et d'autres milieux (humanitaires, formateurs). Il s'agit d'ateliers de trois jours avec cinq à six participants et des encadrants, une démarche intensive. Assal expliquant le chemin qui l'a mené à cette aventure interdisciplinaire: "Chez le médecin surchargé qui s'occupe de diabète et voit chez ses patients toutes les complications de la maladie, il arrive un moment où à force d'être dans le processus de réparation, un travail de Sisyphe, tu entres dans une routine harassante.» D'où le besoin de rechercher des manières d'impliquer activement le malade. Comme beaucoup de novateurs: «Lorsque nous avons démarré, j'ai rencontré une résistance de la part des collègues: 'La médecine c'est la médecine, le théâtre c'est un divertissement!' J'étais parti à contre-courant, pour faire comprendre le rôle du théâtre dans sa résonance avec le vécu des patients, [même si,] dans un univers formé et parfois déformé par des cascades d'algorithmes, l'approche théâtrale a quelque chose d'antinomique.»

Dans l'introduction: "Le Théâtre du Vécu n'est pas 'que' du théâtre, ni 'que' de la médecine ou de l'éducation. Il emprunte à ces domaines mais se distingue par ses finalités. Son pari est qu'en écrivant puis en mettant en scène son vécu, chacun peut surmonter et reprendre en main un nœud biographique ou une impasse dans son parcours de vie.» Il importe de souligner qu'il ne s'agit pas d'improviser à la va-vite des saynètes... mais bien d'entreprendre une démarche rigoureuse, avec des règles précises - d'où la nécessité d'un encadrement professionnel (par des gens de théâtre). "Les patients qui participent au Théâtre du Vécu n'ont sou-

\section{En mettant en scène son vécu, chacun peut reprendre en main une impasse dans son parcours de vie.}

vent jamais rien écrit de leur vie; leurs messages sont maladroits, voire ambigus, mais ils ont une force brute et sauvage - dans ce sens, je les vois comme de petits Shakespeare.»

Noter que, depuis les débuts, Olivier Horn, auteur de films documentaires, a réalisé avec Jean-Philippe Assal plusieurs films, dont des séquences constituent le DVD joint au livre. Quelle différence entre participer au Théâtre du Vécu et consulter un psychologue? Réponse d'une femme de théâtre et formatrice: «Le processus créateur. On permet à la personne de s'exprimer, avec les mots qu'elle veut. On ne juge pas si sa direction est bonne ou mauvaise. Cela permet de se reconnecter avec la partie créatrice en chacun de nous. Il y a aussi quelque chose de fort dans l'œuvre d'art quand elle est partagée, le courage de montrer qui on est, face aux autres.»

Cet ouvrage fourmille de vignettes cliniques et d'observations du déroulement des sessions, il inclut de nombreuses élaborations théoriques et indications pratiques. C'est là une description fort intéressante et complète d'une démarche qui ajoute à la panoplie des moyens développés afin de permettre aux malades chroniques de mieux gérer leur affection et son évolution. 